\title{
Experimental research on the sound recognition of the electric vehicle motor
}

\author{
Dongmei $\mathrm{Hu}^{1}$, Peiyun $\mathrm{Xu}^{3}$, Cheng $\mathrm{Cao}^{1}$, Xiaojun $\mathrm{Yan}^{1}$, Zhihong $\mathrm{Hu}^{2}$, Zhihuan $\mathrm{Song}^{2}$ and \\ Huibin $\mathrm{Li}^{3, *}$ \\ ${ }^{1}$ Acoustic center, SAIC Volkswagen Automotive Company, Co., Ltd., No.1199 Yuan Gong Road, \\ Anting, Shanghai, P.R. China \\ ${ }^{2}$ Huayu Automotive Electric Drive System Co., Ltd, No.88, Jinwen Road, Pudong New Area, \\ Shanghai, P.R. China \\ ${ }^{3}$ School of Mechanical and Vehicular Engineering, Beijing Institute of Technology, Beijing 100081
}

Keywords: Electric vehicle motor, Acoustic array, Recognition, Order tracking method.

\begin{abstract}
By synthetically applying order tracking method and acoustic holography method, the noise source of the electric vehicle motor was discerned in the bench test. According to profiling of the test data, the motor noise order tracking diagrams and sound holography contour maps were employed to draw the noise map of the electric vehicle motor. It was revealed that the low and medium frequency components of the motor's noise are mainly related to the rotational passing frequency of the bearing rolling element and cage, as well as the doubling frequencies of the rotational frequencies of the rotor. The medium and high frequency components of motor's noise are mainly related to motor's loads and speeds. In the 1000-2500 r/min speed stage of the motor with load, the main order of motor's noise is the 72nd order and in the speed stage of $3000-5000 \mathrm{r} / \mathrm{min}$, motor's noise is mainly the 15th, 18th and 26th orders. When the motor speed increases from $5000 \mathrm{r} / \mathrm{min}$ to $8000 \mathrm{r} / \mathrm{min}$, the main order of motor's noise is the fourth order. When the motor was accelerated in no-load operation, its noise characteristics are different from those of the motor with load. When the motor run at no load and low speed, the 16th and $72 \mathrm{nd}$ order are the main order of motor noise, while at high speed, the 2nd order of motor noise and the orders of motor switching frequency noise caused by frequency converter are the main order of motor noise. The above conclusions will give the assistance to further research on the acoustic characteristic of the electric vehicle motor.
\end{abstract}

\section{Introduction}

With the increasing demands for reducing the noise of the electric vehicle motor, it is very significant to distinguish the acoustic source of the electric vehicle motor, and then to do studies on sound control principles and means of the electric vehicle motor.

\footnotetext{
**Corresponding author: huibinli@163.com
} 
The frequency of motor's noise is broad and its frequency range is between $185 \mathrm{~Hz}$ and $7000 \mathrm{~Hz}$. In its broadband continuous spectrum, there are frequencies related to electromagnetic vibration noise, mechanical noise and pneumatic noise of the motor. There are few documents related to motor sound source identification and noise control $[1,2,7,8]$. The major methods and technologies for discerning the sound of the electric vehicle motor are currently the order tracking method, acoustic holographic measurement, acoustic array test, acoustic modal analysis and sound intensity measurement [3-6]. In this study, first of all, based on the theory and method of acoustic array, the radiated noise of motor surface will be measured in bench test, and then four points of far-field measurement for the motor will be employed to plot the motor noise order tracking diagrams. Lastly, acoustic holographic images will be used to draw the acoustic amplitude distribution of the motor to help identify the noise source.

\section{Sound array recognition theory}

The sound source with different properties generates special acoustic signals and the sound waves, according to developed laws of physics in space, spread and form the acoustic field. The time course of acoustic pressure can be surveyed at one location in the acoustic field, by employing the non-directional characteristic acoustic sensors. This time course of acoustic noise stands for the air pressure oscillation in the location of the microphones, but this time course cannot contain much more acoustic source messages. Several microphones, fixed up at the specific locations in space, construct a sound array, by which the spatial acoustic field signal is tested, and much acoustic source message would be gained by analyzing the test data. So the acoustic source will also be discerned.

As shown in Fig.1, presuming plane $\beta$ closes to the outer surface of the electric vehicle motor. There are many sounding parts on the motor. Prior to discerning the true and major sound sources, it could be taken into account that the major sound source is generated on this hypothetical plane $\beta$. Under this hypothesis, only the acoustic distribution in this plane $\beta$ is calculated, then the recognition of the motor could be gained.

Presume that at one location $\mathrm{n}$ in the plane $\beta$, there is a spot acoustic source, and the array $\mathrm{H}$ is employed to focus on this location $\mathrm{n}$. If there a sound source exists at the point $\mathrm{n}$, then the phases relationships among tested signals of different microphones, from the sound source at the point $n$, are confirmed. The tested signals of different microphones are analyzed by employing the beamforming approach with the cumulative delay, to gain the sound-field value of the $\mathrm{n}$ location source. Employing the same procedure to process through the entire plane $\beta$, the acoustic field distribution on this plane will be acquired, and assist to discern which parts of the motor having the major radiating sources.

The coordinate of the location $\mathrm{n}$, on the plane $\beta$, is $(\mathrm{xn}, \mathrm{yn}, \mathrm{zn})$; The coordinate of the sensor si, which pertains to the array $\mathrm{H}$, is (xi,yi,zi). Then the space between the acoustic source position $\mathrm{n}$ and the sensor location $\mathrm{n}$ is described as

$$
D_{i n}=\sqrt{\left(x_{n}-x_{i}\right)^{2}+\left(y_{n}-y_{i}\right)^{2}+\left(z_{n}-z_{i}\right)^{2}}
$$

The sound wave at the acoustic source $n$ radiates at time $t$, and then the time of its reaching the sensor si is $\mathrm{t}+\mathrm{Din} / \mathrm{c}$. In the light of the theory of the beamforming method with the cumulative delay, the output by aiming at the acoustic source point $n$, with the array $\mathrm{H}$, is described as 


$$
p_{n}=\frac{1}{k} \sum_{i=1}^{k} \frac{D_{i n}}{D_{r e f}} g_{i} p_{i}\left(t+\frac{D_{i n}}{c}\right)
$$

where $D_{\text {ref }}$ is space between the plane $\beta$ and the reference sensor location, $c$ is the sound velocity, $k$ is the number of total microphones; $g_{i}$ is the window function of the array, which can be utilized to lower effectively the sidelobe height of the beamforming.

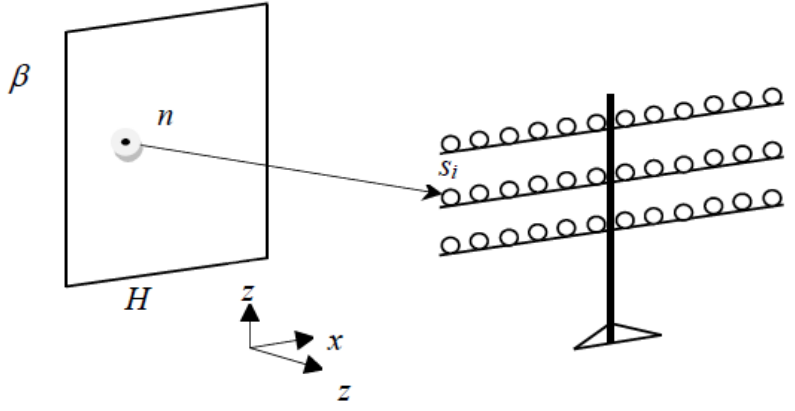

Fig. 1. Schematic diagram of recognition for sensor array.

The plane $\beta$ is divided into $\mathrm{N}$ discrete points of acoustic source, and the calculation of each point of the acoustic source is conducted, in the light of the algorithm introduced above. Scanning the entre plane $\beta$, the acoustic field distribution will be acquired, and the major source of motor will be discerned.

\section{Bench experiment for discerning the acoustic source of the electric vehicle motor}

\subsection{Test system}

As shown in Fig.2, the experimental system comprises the signal acquisition and analysis system, such as LMS TEST Lab9A, SC310DC-UTP, and acoustic sensors. The sensor model is AWA14604, its frequency response range of $20 \mathrm{~Hz}-20 \mathrm{kHz}$, and its sensitivity of $50 \mathrm{mv} / \mathrm{Pa}(250 \mathrm{~Hz})$.

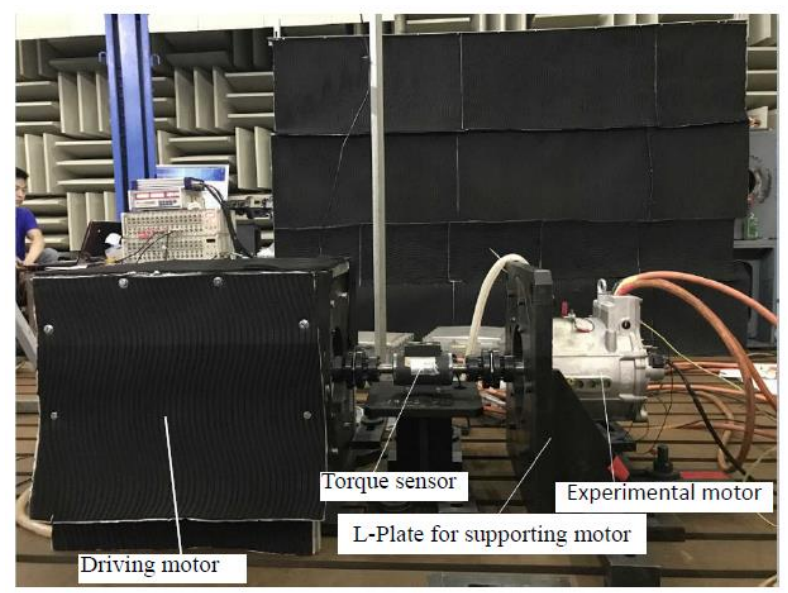

Fig. 2. Layout for the acoustic recognition of motor. 


\subsection{Layout of the sensor array}

Assume a test plane there when the sound array technique is utilized to discern the sound source. There are altogether 24 sensors and three lines of sensors fixed up, and each line includes eight microphones. The space between the microphone plane and the assumed plane is $1500 \mathrm{~mm}$, the interval between lines is $125 \mathrm{~mm}$, and the space between adjacent microphones is $50 \mathrm{~mm}$. The reference point is apart from the assumed plane $50 \mathrm{~mm}$.

\subsection{Test results}

On the base of the characteristic curves and working speeds of the electric vehicle motor, the experimental rotating speed was from $1000 \mathrm{r} / \mathrm{min}$ to $8000 \mathrm{r} / \mathrm{min}$. Fig.3 is the chart reflecting four points of far-field test for the motor. Fig. 4 is the experimental results by using four points of far-field measurement for the motor. Fig.5 is the motor noise order tracking diagram when the motor is accelerated from 1000r/min to 5000r/min. Fig.6 and Fig.7 are acoustic source recognition holograms by utilizing the microphone array technique.

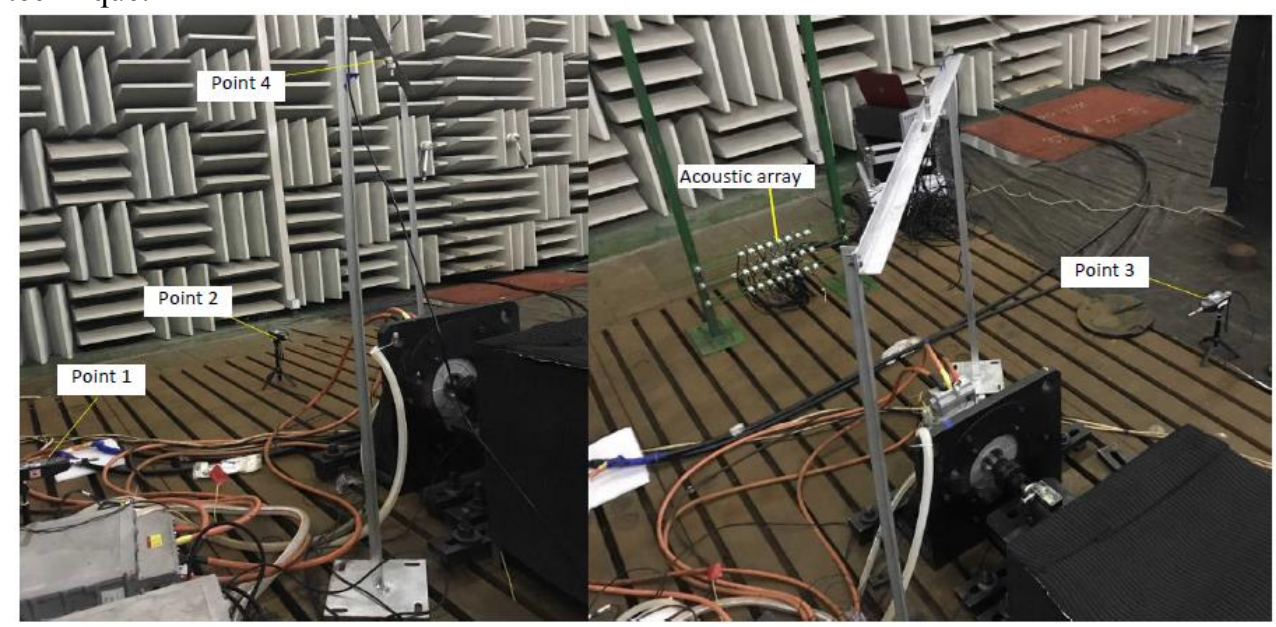

Fig. 3. Four points of far-field microphone test for the electric vehicle motor.

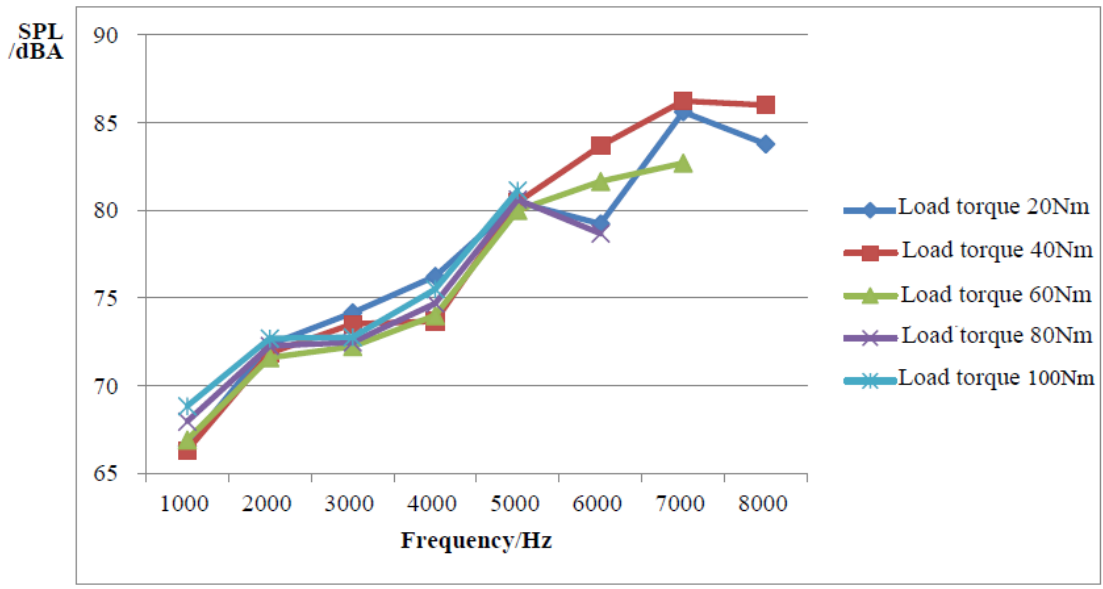

Fig. 4. A-weighted average sound pressure level of motor noise based on four points of acoustical sensors 


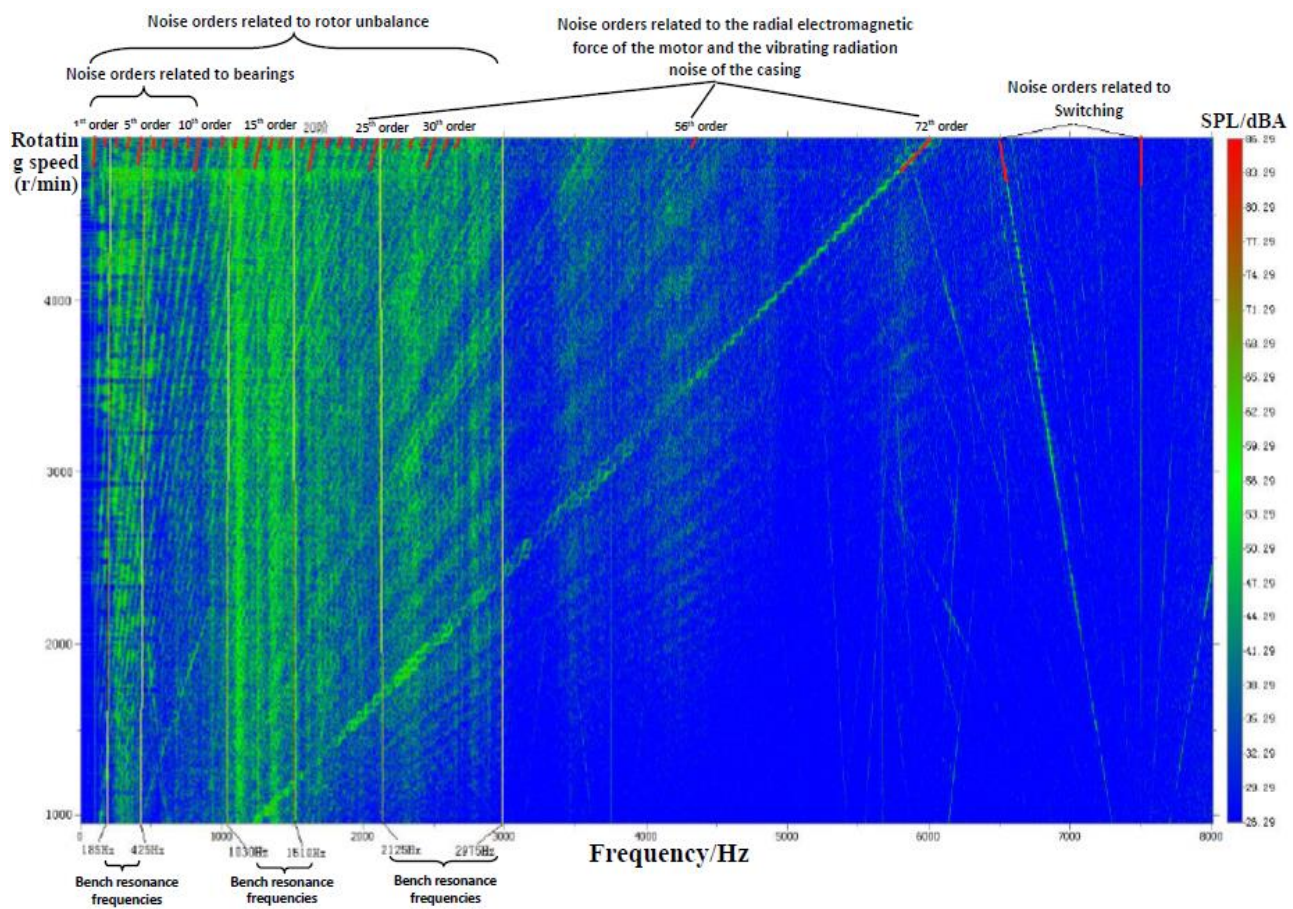

Fig. 5. Motor noise order tracking diagram during the motor accelerated from $1000 \mathrm{r} / \mathrm{min}$ to $5000 \mathrm{r} / \mathrm{min}$.
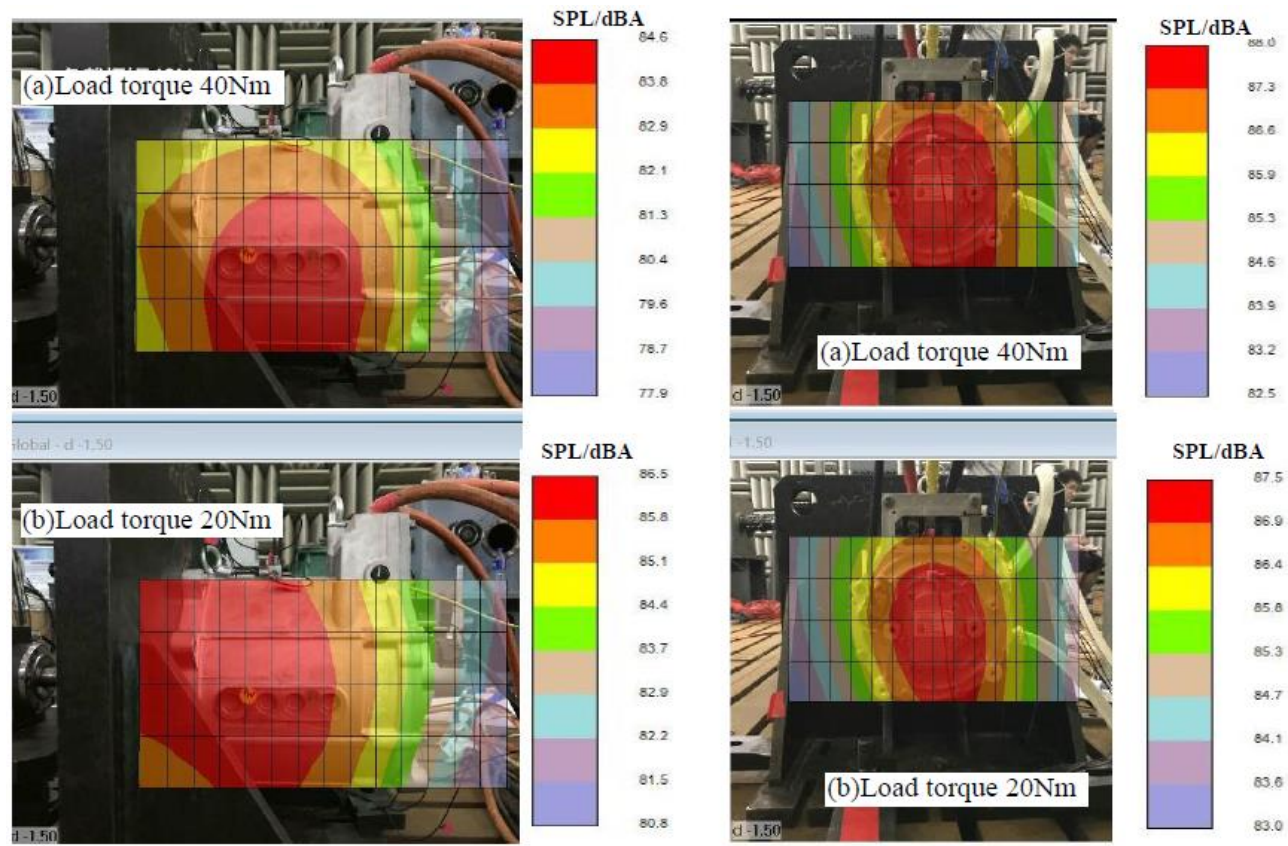

SPL/dBA
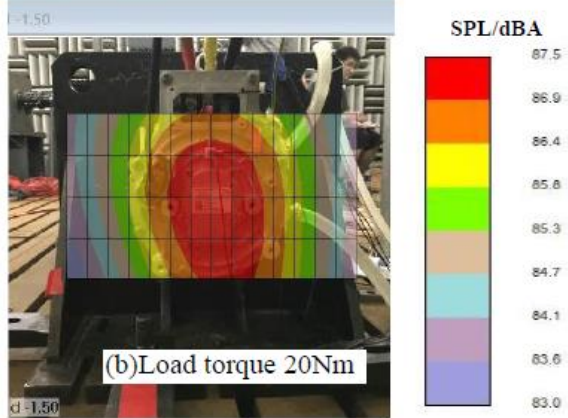

Fig. 6. Acoustic hologram of motor at $8,000 \mathrm{r} / \mathrm{min}$.

Fig. 7. Acoustic hologram of motor at $8,000 \mathrm{r} / \mathrm{min}$ while acoustic arrays positively aligned with motor end faces. 


\subsection{Test Results' Analysis}

(1) The average noise measurement results of four sound level meters at the center of rectangular envelope of motor show that the average sound pressure level of the motor's noise increases as the motor speed increases. When the motor's rotating speed is $7000 \mathrm{r} / \mathrm{min}$ and the load torque is $40 \mathrm{Nm}$, the average sound pressure level reaches the maximum value of $86.23 \mathrm{dBA}$. The cause of this phenomenon may be that the test rig has resonated at this rotating speed.

(2) When the motor is accelerated from $1000 \mathrm{r} / \mathrm{min}$ to $8000 \mathrm{r} / \mathrm{min}$, the order tracking analysis of motor test noise shows that the motor's noise is closely related to the mechanical noise of the rotor bearing, the unbalanced mechanical noise of the rotor, the radiated noise of electromagnetic radial vibration, the air gap noise and the unreasonable resonance frequency of the bench design.

(3) Acoustic hologram of motor at different rotating speeds indicates that when the acoustic array is positively aligned with the circumference of the motor, the noise pressure at the junction between the motor and the L-plate is the largest. Noise generation here is mainly due to the following reasons:

1) The connection between the spline shaft of the torque sensor and motor rotor is poor in neutrality, which results in greater noise.

2) The internal support bearing of the motor has low precision, so it produces large noise.

(4) When the sound array is facing the end face of the motor and the motor is running at high speeds, the sound pressure cloud image is elliptical. This pattern is consistent with the rotor axis trajectory shape. Changes in the trajectory of the rotor cause a corresponding change in the electromagnetic vibration radiation of the motor.

\section{Summary}

(1) The noise source of the electric vehicle motor in the bench test can be clearly identified by synthetically applying the order tracking analysis method and acoustic hologram method.

(2) In the 1000-2500 r/min speed stage of the motor with load, the main order of motor's noise is the 72nd order and in the speed stage of 3000-5000 r/min, motor's noise is mainly the 15th, 18th and 26th orders. When the motor speed increases from $5000 \mathrm{r} / \mathrm{min}$ to $8000 \mathrm{r} / \mathrm{min}$, the main order of motor's noise is the fourth order. When the motor was accelerated in no-load operation, its noise characteristics are different from those of the motor with load. When the motor runs at no load and low speed, the 16th and 72nd order are the main order of motor noise, while at high speed, the 2 nd order of motor noise and the order of motor switching frequency noise caused by frequency converter are the main order of motor noise. The above conclusions will be helpful to researches on the vibration and noise of the electric vehicle motor.

(3) The average noise measurement results of four sound level meters at the center of rectangular envelope of motor show that the average sound pressure level of the motor's noise increases as the motor speed increases. When the motor's rotating speed is $7000 \mathrm{r} / \mathrm{min}$ and the load torque is $40 \mathrm{Nm}$, the average sound pressure level reaches the maximum value of $86.23 \mathrm{dBA}$.

(4) Acoustic hologram of motor at different rotating speeds indicates that when the acoustic array is positively aligned with the circumference of the motor, the noise pressure at the junction between the motor and the L-plate is the largest. The connection between the spline shaft of the torque sensor and motor rotor is poor in neutrality, which results in greater noise. 
(5) When the sound array is facing the end face of the motor and the motor is running at high speeds, the sound pressure cloud image is elliptical. Changes in the trajectory of the rotor cause a corresponding change in the electromagnetic vibration radiation of the motor.

The authors express their gratitude to the SAIC Industrial Foundation Fund (1729) for the financial support of the study.

\section{References}

1. Guan Lili. Noise source identification and optimization of permanent magnet synchronous motor. Proceedings of the 14th Henan Auto Engineering Science and Technology Symposium, 2018, pp.380-382. ( in Chinese)

2. Lai Jianbin. Noise analysis and experimental study on electric vehicle driven motor. A Dissertation Submitted for the Degree of Master of Hefei University of Technology, 2018. ( in Chinese)

3. Wang Guangping. Application of Order Tracking Analysis in Identification of Electric Vehicle Interior Noise Resource. A Dissertation Submitted for the Degree of Master of Jiangsu University, 2011. ( in Chinese)

4. ZHANG Chengning, WANG Zaizhou, SONG Qiang. Research of noise source identification of traction motor system for electric vehicle based on microphone array. Proceedings of the CSEE, 2008, 28(30):109-112. ( in Chinese)

5. Ko H S, Kim K J. Characterization of noise and vibration sources in interior permanent-magnet brushless DC motors [J], IEEE Transactions on Magnetics, 2004,40(6):3482-3489.

6. Islam R, Husain I. Analytical Model for Predicting Noise and Vibration in Permanent-Magnet Synchronous Motors. IEEE Transactions on Industry Applications, 2010,46(6): 2346-2354. (in Chinese)

7. Song Zhihuan. Research on identification technology of electromagnetic vibration and noise source of permanent magnet synchronous motor (PMSM). A Dissertation Submitted for the Degree of Master of Shenyang University of Technology, 2010. (in Chinese)

8. Huibinli, Mengxi Ning, Lei Hou, Tiangqi, Zhou. Experimental study on the noise identification of the turbocharger. Proceedings of the 3CA2011, 2011, pp.1-7. 